\title{
CORRELATIONS IN MAGNETIC-NONMAGNETIC SEMICONDUCTOR SUPERLATTICES - SIMPLE MODEL
}

\author{
J. BLINOWSKI \\ Institute of Theoretical Physics, Warsaw University \\ Hoża 69, 00-681 Warszawa, Poland \\ AND P. KaCMAN \\ Institute of Physics, Polish Academy of Sciences \\ Al. Lotników 32/46, 02-668 Warszawa, Poland
}

\begin{abstract}
The magnetic interactions in superlattices formed by alternating layers of magnetic and nonmagnetic semiconductors with common anions were studied theoretically. Within a one-dimensional tight-binding model by minimising the total electronic energy we show the existence of an efficient long range mechanism of magnetic correlations between the neighbouring magnetic layers in such superlattices. The cases of magnetic ions in the barriers (e.g., $\mathrm{EuTe} / \mathrm{PbTe}$ ) and in the wells, with the order within the magnetic layers being either ferromagnetic or antiferromagnetic, have been considered and compared with the paramagnetic case. In the case of antiferromagnetic ordering within magnetic layers we have found that for even and odd numbers of magnetic monolayers different magnetic superlattices are energetically favourable.
\end{abstract}

PACS numbers: 75.70.Ak, 75.25.+z

The magnetic correlations between the ferromagnetic metallic layers separated by nonmagnetic metals and insulators have been widely studied for the last ten years, both experimentally and theoretically. In the case of the metallic spacers several models have been proposed to explain the correlation mechanism: the RKKY model, the free electron model, the tight-binding model with spin dependent potential steps, etc. For the insulating spacers the model of electron tunnelling has been advocated (for references see, e.g., [1]). However, it has been shown by Bruno [1] that there is a common feature of all these models, which is the spin dependent change of the density of states resulting from the quantum interference of the free electron waves.

Recently, the magnetic correlations were also discovered in semiconductor superlattices - the neutron diffraction studies of EuTe/ $\mathrm{PbTe}$ superlattices showed 
satellite peaks, which proved the existence of magnetic order extending over many superlattice periods. The correlations between the spin directions in the successive magnetic barriers were shown to persist even when the magnetic layers were separated by thick (up to 20 nonmagnetic monolayers) spacers [2]. Similar correlations were also observed in $\mathrm{MnTe} / \mathrm{CdTe}$, more complicated superstructures with helical spin ordering [3].

The EuTe is an antiferromagnet with the Neel temperature $T_{\mathrm{N}}=9.6 \mathrm{~K}$. The AFM-II spin ordering that forms in EuTe below the Néel point consists of ferromagnetic "sheets" of spin on (111)-type planes, which are antiferromagnetically coupled to one another [4]. In (111) EuTe/PbTe superlattices the AFM-II structure is preserved in the EuTe layers and the ferromagnetic sheets always form on the (111) planes, i.e., they are perpendicular to the superlattice growth axis. Both $\mathrm{EuTe}$ and $\mathrm{PbTe}$ are semiconductor compounds. In the temperature region below the $T_{\mathrm{N}}$, EuTe, with a wide energy gap of $2 \mathrm{eV}$, is an insulator. PbTe is a nonmagnetic semiconductor with a relatively narrow gap $(\approx 0.2 \mathrm{eV})$. Thus, the origin of the observed in these superlattices long-range magnetic correlations cannot be understood on the grounds of the existing theories of interlayer coupling, because in all the above-mentioned models the high concentrations of conduction electrons play a crucial role and, moreover, these models were derived for a ferromagnetic order within the magnetic layers.

In this work we prove that the quantum interference between the spin dependent perturbations in successive barriers is an effective mechanism for magnetic long range correlations also in the case of semiconductor superlattices with the antiferromagnetic ordering in the magnetic layers. We consider a one-dimensional tight-binding model with every atom in the one-dimensional chain representing an entire atomic monolayer. The $m$ magnetic cations with the on-site energies $e_{m}$ are situated, like for the experimentally studied $\mathrm{EuTe} / \mathrm{PbTe}$ superlattices, in the barriers whereas the $n$ nonmagnetic cations, with the on-site energies $e_{n}$, in the wells of the superlattice. The on-site anion energies are $e_{\mathrm{A}}$. We limit the nonmagnetic interatomic interactions to the nearest-neighbour anion-cation coupling constant $a$. We assume that the electrons occupying magnetic cation sites interact via direct exchange, characterised by the exchange constant $J$, with the localised spins of the magnetic cations. In addition, we allow for a weaker, kinetic-type exchange, described by an exchange constant $J_{\mathrm{A}}$, between the electrons in the anion orbitals and the spins of the nearest magnetic cations.

For given values of $m$ and $n$ we calculate the band structure in the entire one-dimensional Brillouin zone for two different cases: (i) with identical spin configurations in successive magnetic layers and (ii) with successive magnetic layers having reversed spin configurations. The spin configuration, which leads to the lower total electronic energy is, most probably, the one chosen by the magnetic superlattice. The difference $\Delta E(m, n)$ of the total electronic energy between the two cases is a measure of the strength of the interlayer magnetic coupling, which correlates the Eu spins across the nonmagnetic layer.

In our model, the Bloch functions for the occupied valence bands and the empty conduction bands are linear combinations of the tight-binding functions constructed from cation and anion orbitals. We limit the number of orbitals to one 
orbital $\varphi_{\alpha}(r)$ per atom

$$
\psi_{\alpha}(x)=\frac{1}{\sqrt{N}} \sum_{i=1}^{N} \exp \left[\mathrm{i} k\left(\boldsymbol{R}_{i}+t_{\alpha}\right)\right] \varphi_{\alpha}\left(\boldsymbol{r}-\boldsymbol{R}_{i}-\boldsymbol{t}_{\alpha}\right),
$$

where $N$ is the number of the elementary superlattice cells, each consisting of $2 m$ magnetic cations, $2 n$ nonmagnetic cations and $2 n+2 m$ anions. $\alpha$ runs from 1 to $4 m+4 n$. The odd values of $\alpha$ correspond to anions. The even values of $\alpha$ from $\alpha=2$ to $\alpha=2 m$ and from $\alpha=2 m+2 n+2$ to $\alpha=4 m+4 n$ denote the magnetic cations and the remaining even values of $\alpha$ denote the nonmagnetic cations. Vectors $t_{\alpha}$ describe the positions of the atoms in the elementary superlattice cell. Note that the big elementary cell, consisting of $4 n+4 m$ atoms, is necessary for the case of the opposite spins in the successive magnetic layers, whereas in the other case, i.e., the case with identical spin orientations in all magnetic layers, it is enough to introduce an elementary superlattice cell consisting of $2 n+2 m$ atoms.

In the space of the Bloch functions $\psi_{\alpha}(r)$, the one-dimensional crystal Hamiltonian is represented by a $(4 m+4 n) \times(4 m+4 n)$ matrix and the band structure is obtained by diagonalization of this matrix. We calculate $\Delta E(m, n)$ for superlattices consisting of antiferromagnetically ordered spins of the magnetic atoms situated in the barriers. The one-dimensional model is not suited to represent quantitatively any real three-dimensional superlattice, still we chose the parameters as to reproduce the energy gaps and the width of the bands of PbTe and EuTe $\left(e_{n}=0, e_{m}=1.8 \mathrm{eV}, e_{\mathrm{A}}=-0.2 \mathrm{eV}, a=1.5 \mathrm{eV}\right)$. Also the values $J=0.35 \mathrm{eV}$ and $J_{\mathrm{A}}=0.08 \mathrm{eV}$ are chosen to be of the order expected for the direct and kinetic exchange for $f$-shell atoms in $\mathrm{PbTe}$ [6]. The essential features of the obtained results are as follows:

(1) The spin-spin interactions lead to the lowering of the total energy of the system (the total electronic energy obtained by putting $J=J_{\mathrm{A}}=0$ is always higher).

(2) For even $m$ values the lower energy is obtained for identical magnetic structures in consecutive barriers, whereas for odd values of $m$ the opposite spin directions in neighbouring barriers are energetically favoured. This means that whatever the total magnetic moment of the single barrier is (i.e., zero as for even $m$ or finite as for odd $m$ ) the lower energy corresponds to the superlattice with zero average magnetic moment, in agreement with the results of SQUID measurements of magnetization and magnetic susceptibility of $\mathrm{EuTe} / \mathrm{PbTe}$ superlattices [7].

(3) The absolute value of $\Delta E(m, n)$ is a monotonically, but slowly decreasing function of the spacer thickness $n$ (see Fig. 1). Even for the largest considered spacer thicknesses the correlation energy $\Delta E(m, n)$ is of the same order of magnitude as the correlation energy per one surface atom measured in metallic, ferromagnetic layers [8].

(4) Surprisingly enough, apart from the few lowest values of $m$, for a given number of nonmagnetic monolayers $n$ the results are essentially independent of the number of magnetic monolayers $m$. This seems to indicate that the magnetic correlations depend primarily on the relative orientations of the spins at the two interfaces of the nonmagnetic spacer, the lower energy corresponds always to the opposite directions of these spins. This conclusion is also confirmed by the results 


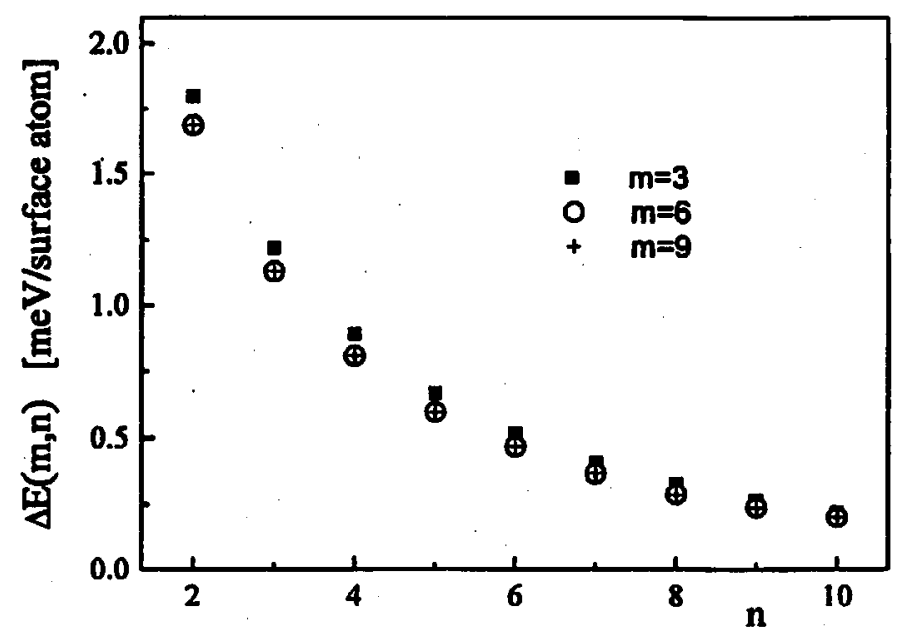

Fig. 1. The dependence of the magnitude of correlation energy $|\Delta E(m, n)|$ on the spacer thickness $n$ for three different thicknesses of the magnetic layer: $m=3,6,9$ monolayers.

obtained by switching off the kinetic exchange, i.e., the interaction effective only at the interfaces, putting $J_{\mathrm{A}}=0$ diminishes the correlation energy roughly by factor 3 .

Qualitatively, the same results have been also obtained for superlattices with antiferromagnetically ordered spins placed in the wells separated by nonmagnetic barriers.

Finally, we have allowed for a ferromagnetic alignment of the spins in the magnetic layers in the barriers. This case corresponds to the situation in the recently fabricated $\mathrm{EuS} / \mathrm{PbS}$ superlattices [9]. In contrast to the results for the antiferromagnetic layers presented above, in all studied superlattices ( $m=3,4$; $n=2-6$ ) with the ferromagnetic order within the magnetic barriers the same spin orientation in the consecutive magnetic layers was energetically favoured in our model. This should lead to a finite macroscopic magnetic moment in the ferromagnetic semiconductor superlattices. The magnetization of the EuS/PbS superstructures, measured in Ref. [9] by using a SQUID magnetometer, seems to confirm this result.

This work was partially supported by the Committee for Scientific Research grant No. 2 P03B 02511.

\section{References}

[1] P. Bruno, Phys. Rev. B 52, 411 (1995).

[2] T.M. Giebułtowicz, V. Nunez, G. Springholz, G. Bauer, J.J. Chen, M.S. Dresselhaus, J.K. Furdyna, J. Magn. Magn/Mater. 140-144, 635 (1995).

[3] V. Nunez, T.M. Giebułtowicz, W. Faschinger, G. Bauer, H. Sitter, J.K. Furdyna, J. Magn. Magn. Mater. 140-144, 633 (1995). 
[4] P. Wachter, in: Handbook of Physics and Chemistry of Rare Earths, Eds. K.A. Gschneider, L. Eyring, North-Holland Publishing Co., Amsterdam 1979.

[5] V. Nunez, G. Springholz, T.M. Giebułtowicz, H. Kepa, K.I. Goldman, C.F. Majkrzak, G. Bauer, Superlattices Microstruct., in press.

[6] T. Dietl, C. Śliwa, G. Bauer, H. Pascher, Phys. Rev. B 49, 2230 (1994).

[7] Z. Wilamowski, G. Springholz, V. Svrcek, W. Jantsch, Acta Phys. Pol. A 92, (1997), Proc. of this Conf. (Part II); H. Krenn, private communication.

[8] S.S.P. Parkin, Phys. Rev. Lett. 67, 3598 (1991).

[9] A. Stachow-Wójcik, A. Twardowski, T. Story, W. Dobrowolski, E. Grodzicka, A. Sipatow, Acta Phys. Pol. A 92, (1997), Proc. of this Conf. (Part II). 